ARTICLE

\title{
Implementation of a Forced Collision Method in the Estimation of Deposit Energy Distribution with the PHITS Code
}

\author{
Shin-ichiro ABE ${ }^{1, *}$, Yukinobu WATANABE ${ }^{1}$, Koji NIITA ${ }^{2}$ and Yukio SAKAMOTO ${ }^{3}$ \\ ${ }^{1}$ Department of Advanced Energy Engineering Science, Kyushu University, 6-1 Kasuga-koen, Kasuga-shi, Fukuoka, 816-8580, Japan \\ ${ }^{2}$ Research Organization for Information Science and Technology, 2-4 Shirakata-shirone, Tokai-mura, Naka-gun, Ibaraki, 319-1106, Japan \\ ${ }^{3}$ Japan Atomic Energy Agency, 2-4 Shirakata-shirone, Tokai-mura, Naka-gun, Ibaraki, 319-1195, Japan
}

\begin{abstract}
To improve the computational efficiency of the deposit energy calculation in a very small volume due to nuclear reactions, we have attempted to implement a forced collision method in the deposit energy distribution tally of the PHITS (Particle and Heavy Ion Transport code System). The local deposit energy distribution by 100-MeV neutrons penetrating a cubic silicon cell with $\mu \mathrm{m}$ size was calculated by the modified PHITS code. It was found that the computational efficiency was improved in inverse proportion to the cell size. In the case of a 1- $\mu \mathrm{m}$ silicon cell, the forced collision method brought improvement to the computational efficiency by a factor of about 15 . This indicates that the forced collision method is effective to estimate the local deposit energy distribution due to nuclear reactions in a microscopic volume.
\end{abstract}

KEYWORDS: PHITS, radiation transport, energy deposition, variance reduction, forced collision method

\section{Introduction}

In prediction of soft error rates in semiconductor devices and dose estimations in radiation therapy, it is required to calculate with high accuracy the deposit energy distribution (or pulse height distribution) in a small volume by radiation. The authors of Kyushu University have been investigating cosmic-ray neutron induced soft errors, ${ }^{1)}$ using the Particle and Heavy Ion Transport code System ${ }^{2)}$ (PHITS code). The terrestrial cosmic-ray neutrons induced soft error is known as one of the key reliability issues for modern microelectronic devices, and is required to estimate soft error rates by simulations. ${ }^{3)}$ But it takes very long computation time to obtain convergent result because the probability of neutron-induced reactions is extremely small in a micrometric volume size. For such cases, one expects that a forced collision (FC) method is effective as one of the variance reduction schemes.

Unfortunately, the use of the FC method is not allowed in the calculations of deposit energy distribution with the latest version of the PHITS code. Thus, we have attempted to implement the FC method in the deposit energy distribution tally of PHITS on the basis of the deconvolution method. ${ }^{4,5)}$

\section{Forced Collision Method}

The FC method is one of the variance reduction schemes that increases the sampling of collisions in a specified cell. When a specified particle enters a region defined as the FC region, the incident particle splits into uncollided and collided particles as shown schematically in Fig. 1. The uncollided particle passes through the current cell without

*Corresponding author, E-mail: abe@aees.kyushu-u.ac.jp

(C) 2011 Atomic Energy Society of Japan, All Rights Reserved.

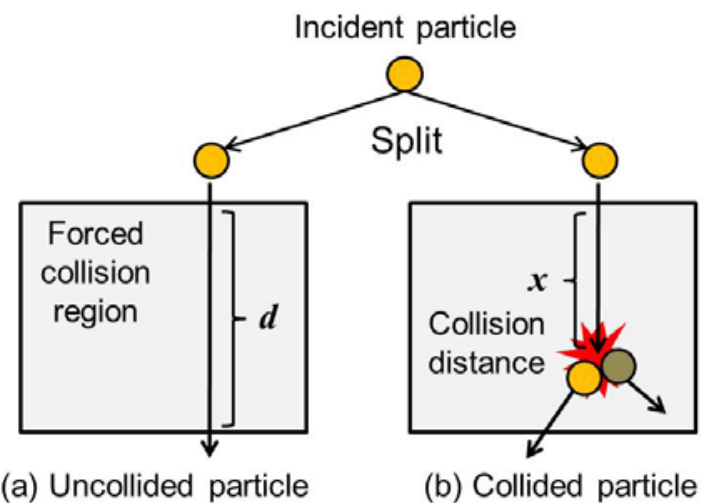

Fig. 1 Schematic interpretation of the FC method

collision. The collided particle is forced to collide within the specified cell. It means that the "passing-through case" and the "collision case" are analyzed due to a single incident particle simulation. These split particles are weighted by the following passing-through or collision probabilities:

$$
\begin{aligned}
& W_{\text {uncoll }}=W_{0} \exp \left(-\Sigma_{t} d\right), \\
& W_{\text {coll }}=W_{0}\left\{1-\exp \left(-\Sigma_{t} d\right)\right\},
\end{aligned}
$$

where $W_{0}, W_{\text {uncoll }}$ and $W_{\text {coll }}$ are the weights of the incident particle, the uncollided particle and the collided particle, respectively. $d$ is the distance to cell surface in the particle's direction, and $\Sigma_{t}$ is the macroscopic total cross section of the cell material. The collision distance $x$ is sampled by the following method. The probability of colliding within a distance $x$ is given by

$$
P(x)=1-\exp \left(-\Sigma_{t} x\right) .
$$




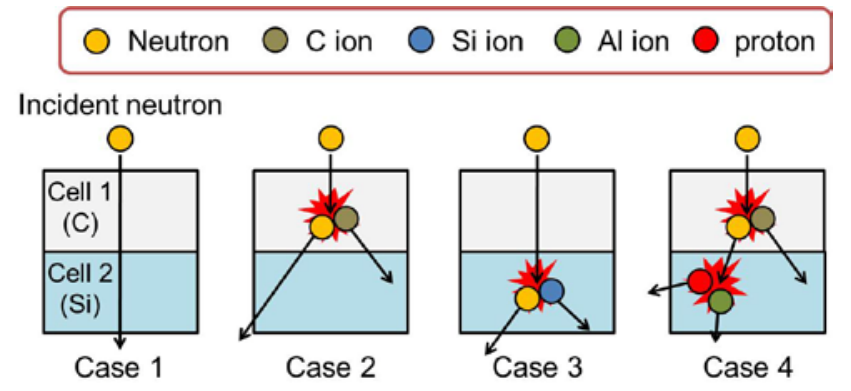

Fig. 2 Classification of each case to be analyzed in a simulation of single neutron incidence on carbon and silicon cells

Whenever the FC method is applied, the collision is assumed to occur within a distance $d$. Thus the position $x$ should be sampled in the interval $0<x<d$ within the cell according to $\xi=P(x) / P(d)$, where $P(x) / P(d)$ is the conditional probability of collision within the distance $x$ and $\xi$ is the random number. By solving Eq. (3) with respect to $x$, the following equation is obtained:

$$
x=-\frac{1}{\Sigma_{t}} \ln \left[1-\xi\left\{1-\exp \left(-\Sigma_{t} d\right)\right\}\right]
$$

If the FC method is applied more than once in a single incident particle simulation, many "cases" should be analyzed. Let us consider an example of neutron-induced events as shown in Fig. 2. The target is composed of a carbon cell (cell 1) and a silicon cell (cell 2), and both cells are defined as FC regions. Neutrons are specified as FC particles. In this example, four cases are analyzed as shown in Fig. 2:

Case 1: Incident neutron passes through both of the cell 1 and the cell 2.

Case 2: Incident neutron collides in the cell 1 and secondary neutron passes through the cell 2.

Case 3: Incident neutron passes through the cell 1 and collides in the cell 2.

Case 4: Incident neutron collides in the cell 1 and secondary neutron collides in the cell 2.

The deposit energy tally differs from standard Monte Carlo tallies (e.g., product tally) in that the deposit energy can only be tallied when the transport of all the particles is finished. In the present work, we incorporate new additional routines based on the deconvolution method ${ }^{4,5)}$ in order to handle the information belonging to each case in the deposit energy tally of the PHITS code.

\section{Deposit Energy Distribution Tally with Group Tree}

To tally deposit energy for each case, the "group number" is allocated tentatively to all particles and "group tree" is formed. Figure 3 shows the group tree of the above example.

First, the incident particle is allocated the "mother group". If the splitting occurs by the FC method, new groups are created, and uncollided and collided particles are allocated separately. The particles generated by a collision belong to

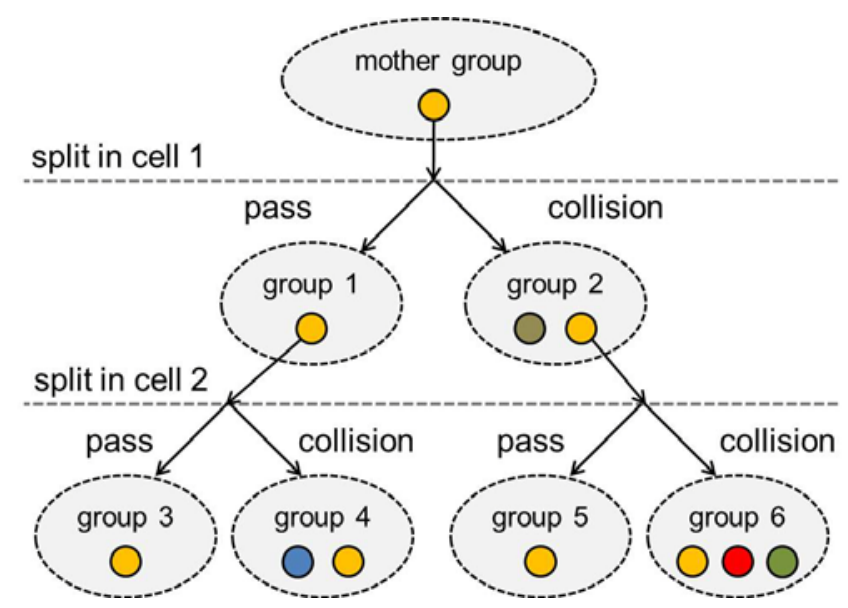

Fig. 3 A scheme of "group tree" caused by forced collisions

the same group as collided particle. The deposit energy and the weight of each group are calculated and temporarily stored. About above example, stored data are as follows:

$$
\begin{array}{ll}
E_{\text {mother }}=0, & W_{\text {mother }}=W_{0} \\
E_{1}=0, & W_{1}=W_{\text {mother }} \exp \left(-\Sigma_{1} d_{1}\right) \\
E_{2}=e_{C}, & W_{2}=W_{\text {mother }}\left[1-\exp \left(-\Sigma_{1} d_{1}\right)\right] \\
E_{3}=0, & W_{3}=W_{1} \exp \left(-\Sigma_{2} d_{2}\right) \\
E_{4}=e_{S i}, & W_{4}=W_{1}\left[1-\exp \left(-\Sigma_{2} d_{2}\right)\right] \\
E_{5}=0, & W_{5}=W_{2} \exp \left(-\Sigma_{2} d_{2}\right) \\
E_{6}=e_{A l}+e_{p}, & W_{6}=W_{2}\left[1-\exp \left(-\Sigma_{2} d_{2}\right)\right]
\end{array}
$$

where $W_{i}$ and $E_{i}$ are the weight and deposit energy of $\boldsymbol{i}$-th group, $e_{C}, e_{S i}, e_{A l}$ and $e_{p}$ are the deposit energy by each ion, $\Sigma_{j}$ is the macroscopic neutron total cross section of the $j$-th cell material and $d_{j}$ is the distance to $j$-th cell surface in the neutron's direction.

After transport of all particles is finished, each case is identified by referring to the group tree and the deposit energy of each case is tallied. The final result of the above example is given according to "case":

Case 1: mother group + group $1+$ group 3

$$
E=0 ; W=W_{3}
$$

Case 2: mother group + group $2+$ group 5

$$
E=E_{2} ; W=W_{5}
$$

Case 3: mother group + group $1+$ group 4

$$
E=E_{4} ; W=W_{4}
$$

Case 4: mother group + group $2+$ group 6

$$
E=E_{2}+E_{6} ; W=W_{6}
$$

This deconvolution method can be applied even if the target is constructed by many cells.

\section{Results and Discussion}

First, the deposit energy distribution in a cubic silicon cell (cell size: 1, 2, 5 and $10 \mu \mathrm{m}$ ) due to $100-\mathrm{MeV}$ neutrons is calculated to validate the modified PHITS code with the FC 


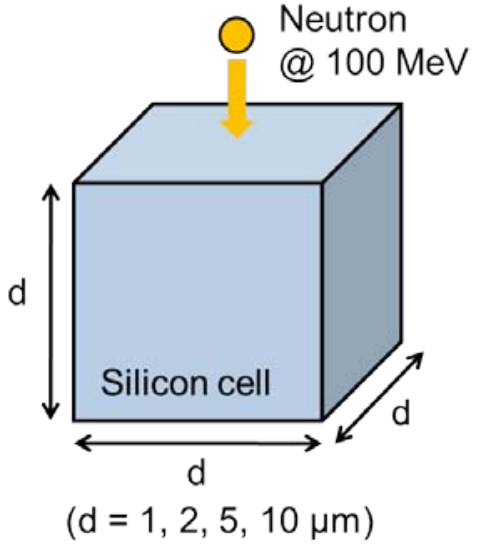

Fig. 4 Neutron incident on silicon cell (cell size: 1, 2, 5, and $10 \mu \mathrm{m})$

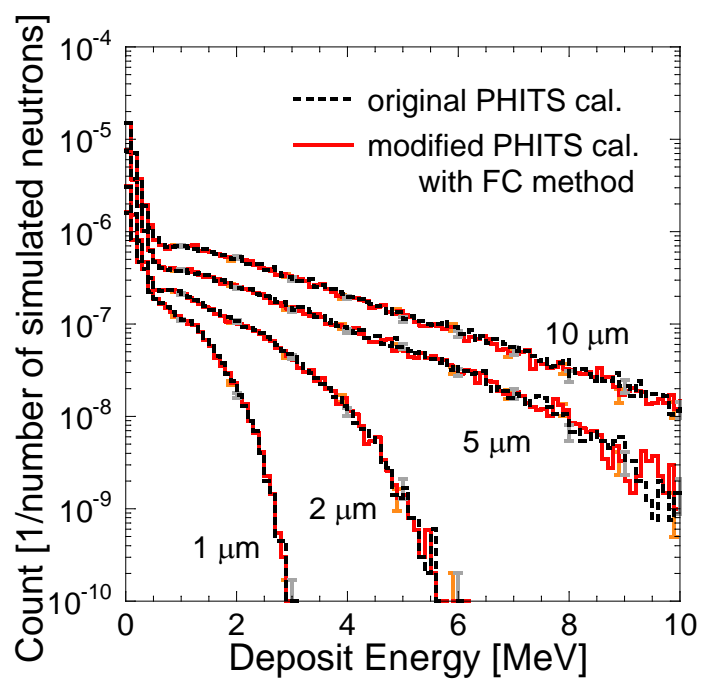

Fig. 5 Comparisons of deposit energy distribution simulated by the PHITS calculations with and without the FC method

method. Figure 4 represents the computational system, and Fig. 5 shows comparisons of the deposit energy distributions calculated by the original PHITS code without the FC method and the modified PHITS code with the FC method. The modified PHITS calculation is in excellent agreement with the original PHITS calculation within the statistical error. This result indicates that the additional routines work well.

Next, computational efficiencies of the FC method in the above simulations are estimated. The computational efficiency is defined by

$$
\text { Efficiency }=\frac{t_{\text {original }}}{t_{F C}} \times \frac{\sigma_{\text {original }}^{2}}{\sigma_{F C}^{2}},
$$

where $t$ is the computational time, $\sigma^{2}$ is the variance, and the subscripts, original and FC, stand for the original PHITS calculation and the PHITS calculation with FC method, respectively. The variances in both the calculations are adjusted to have the same value in the present work, thus the computational efficiency corresponds to the ratio of computational time. In Fig 6, the efficiency of each

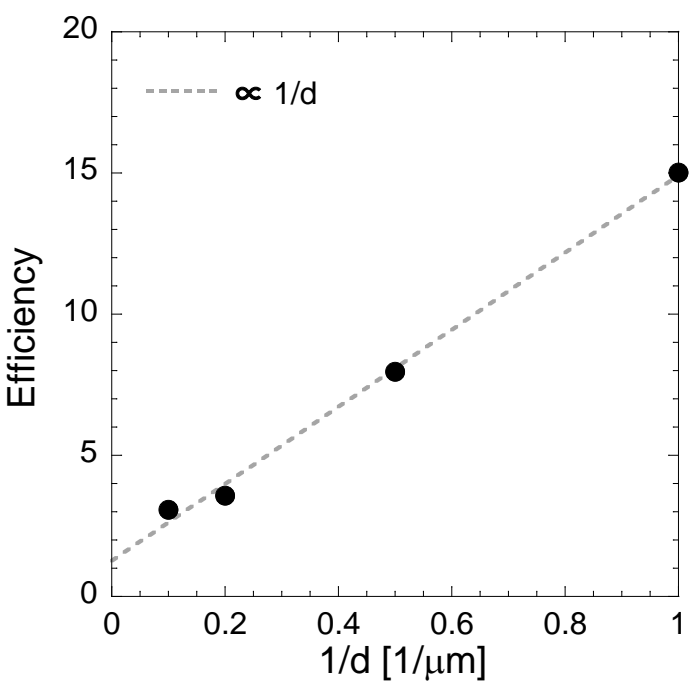

Fig. 6 Computational efficiencies as a function of the reciprocal of the cell size

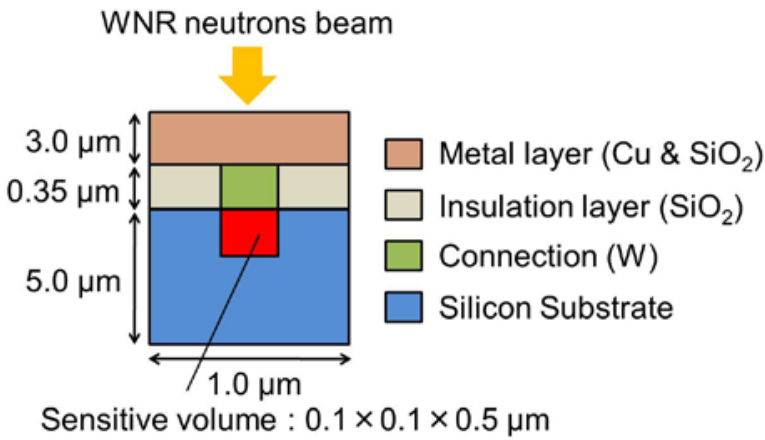

Fig. 7 Schematic structure of the test device

simulation is plotted as a function of the reciprocal of the cell size. It is found that the efficiency is improved in inverse proportion to the cell size. This tendency is attributed to the fact that the first term in Maclaurin expansion of the collision probability, as shown in Eq. (7), is directly proportional to the cell size.

$$
1-\exp \left(-\Sigma_{t} d\right)=\Sigma_{t} d-\frac{1}{2}\left(\Sigma_{t} d\right)^{2}+\frac{1}{6}\left(\Sigma_{t} d\right)^{3} \cdots
$$

In the case of a 1- $\mu \mathrm{m}$ silicon cell, the FC method brought improvement to the computational efficiency, i.e., reduction of the computational time, by a factor of about 15 .

Finally, the modified PHITS code with FC method was applied to a cosmic-ray neutron induced soft error simulation. The configuration of the test device structure is illustrated in Fig. 7. A silicon oxide insulation layer $0.35 \mu \mathrm{m}$ thick is placed on a $1.0 \times 1.0 \times 5.0 \mu \mathrm{m}^{3}$ silicon substrate. A $3.0 \mu \mathrm{m}$ metal layer consisting of copper and silicon oxide is located on an insulation layer and tungsten connects the metal layer and silicon substrate. The entire test device is defined as the FC region, and the deposit energy in a sensitive volume $(0.1$ $\times 0.1 \times 0.5 \mu^{3}$ ) embedded in the silicon substrate is tallied. The incident neutron spectrum is assumed to be the same as that of the Weapons Neutron Research Facility (WNR) neu- 


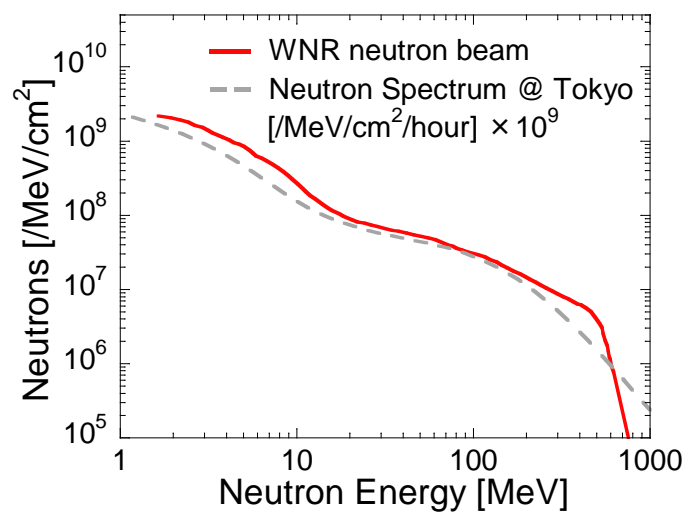

Fig. 8 WNR neutron beam spectrum ${ }^{6}$

tron beam ${ }^{6)}$ shown in Fig. 8, which is similar to a typical terrestrial neutron spectrum observed at the ground level and is widely used for accelerated tests of soft errors.

Figure 9 presents a comparison of the deposit energy distributions. The PHITS calculation with the FC method is in excellent agreement with the original PHITS calculation within the statistical errors except at high energy deposit, where a divergence between both the calculations is seen because of poor statistics. The computational efficiency is improved by a factor of about three, which is equivalent to that of Fig. 6 for $d=8.35 \mu \mathrm{m}$ corresponding to the device length in the incident neutron direction. Thus, the present result indicates that the routines added newly in the deposit energy tally works well even when the target is constructed by several cells and more complicated group tree is formed. Therefore, the FC method is very effective to calculate the local deposit energy distribution caused by neutron-induced reactions in a microscopic volume. Our practical application to soft error simulation has been reported in detail elsewhere. ${ }^{7)}$

\section{Conclusion}

The force collision (FC) method was successfully implemented in the deposit energy distribution tally of the PHITS code. The deposit energy distribution by $100-\mathrm{MeV}$ neutrons incident on a cubic silicon cell with $\mu \mathrm{m}$ size was calculated by the modified PHITS code. It was found that the computational efficiency is improved as the cell size scales down. In the case of a 1- $\mu \mathrm{m}$ silicon cell, the FC method brought improvement to the computational efficiency by a factor of about 15. This indicates that the FC method is useful to estimate the local deposit energy distribution due to nuclear reactions in a microscopic volume with good computational efficiency.

\section{Acknowledgment}

The authors would like to thank N. Sano, Y. Tosaka,

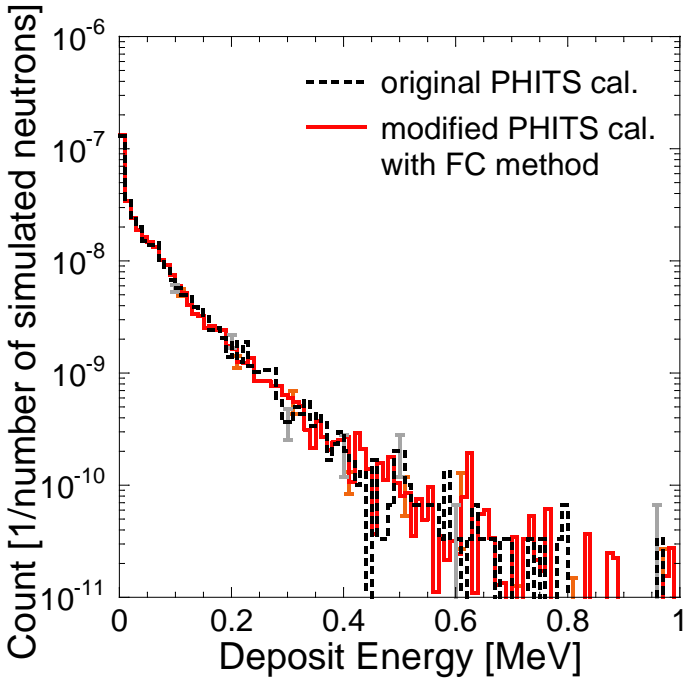

Fig. 9 Comparison of deposit energy distribution between the PHITS calculations with and without the FC method

H. Furuta, M. Tsutsui and T. Imamura for their helpful discussions and comments on soft error simulations.

\section{References}

1) S. Abe, S. Hirayama, Y. Watanabe, N. Sano, Y. Tosaka, M. Tsutsui, H. Furuta, T. Imamura, "Applicability of nuclear reaction models implemented in PHITS to simulations on single-event effects," to be published in Proc. of Int. Conf. on Nuclear Data for Science and Technology (ND2010), Jeju, Korea, April 26-30, 2010.

2) H. Iwase, K. Niita, T. Nakamura, "Development of General-Purpose Particle and Heavy Ion Transport Monte Carlo Code,” J. Nucl. Sci. Technol., 39[11], 1142-1151 (2002).

3) T. Nakamura, M. Baba, Y. Yahagi, H. Kameyama, Terrestrial Neutron-Induced soft Errors in Advance Memory Devices, World Scientific (2008).

4) T. E. Booth, Pulse Height Tally Variance Reduction in MCNP, LA-13955, Los Alamos National Laboratory (LANL) (2002).

5) J. S. Hendricks, G. W. McKinney, "Pulse-Height Tallies with Variance Reduction,” The Monte Carlo Method 2005 Topical Meeting (2005), [CD-ROM].

6) S. A. Wender, S. Balestrini, A. Brown, R. C. Haight, C. M. Laymon, T. M. Lee, P. W. Lisowski, W. McCorkle, R. O. Nelson, W. Parker, N. H. Hill, "A fission ionization detector for neutron flux measurements at a spallation source," Nucl. Instr. Meth. Phys. Res., A336, 226-231 (1993).

7) S. Abe, Y. Watanabe, N. Shibano, N. Sano, T. Arakawa, H. Furuta, M. Tsutsui, T.Uemura, "Simulation of Cosmic-ray Neutron Induced Soft Errors Using PHITS and HyENEXSS", Proc. of The 9th Int. Workshop on Radiation Effects on Semiconductor Devices for Space and Applications (RASEDA-9), Takasaki, Gunma, Japan, October 27-29, 2010, 68-71 (2010). 